\title{
Observing learning and conceptual development through novel product interaction
}

\author{
Christopher Wilkinson \\ Pat Langdon \\ John Clarkson \\ Engineering Design Centre, Department of Engineering, University of Cambridge \\ Trumpington Street, Cambridge CB2 1PZ, UK \\ crw42@eng.cam.ac.uk pm/24@eng.cam.ac.uk \\ pjc10@eng.cam.ac.uk
}

\begin{abstract}
Improving product usability through inclusive design consideration can enhance a products potential commercial success, whilst widening it's acceptability across an increasingly divergent market. An experimental approach was developed to encapsulate how individuals perceive, process and respond to stimuli during interaction with products. By presenting a sample of participants with a novel product, we are able to assess how their understanding and internal conceptualisations are developed during increasing product exposure, and indicate how product design can have a significant impact upon these processes. Participants were recorded interacting with the novel product whilst providing concurrent protocol and information elicited regarding the development of internal representations. The extent of participants' technological familiarity was also investigated to determine how prior experience may assist novel product interaction. Age related differences were evident in both approaches to problem solving and extent of technological familiarity, and this was considered to have an impact upon overall interactional performance.
\end{abstract}

Inclusive Design, Interface Interaction, Prior Experience, Learning, Mental Models.

\section{INTRODUCTION}

The aim of inclusive design is to improve product design and interface interaction for everyone, including the older generation

\begin{abstract}
"an increasing market sector that is regularly neglected. Inclusive design has been defined as: "The design of products and/or services, accessible to and useable by people with the widest range of abilities within the widest range of situations without the need for special adaptation or design." [1]
\end{abstract}

Possessing the commercial potential to increase long-term profits, and enhance manufacturers' competitive edge, inclusive design can assist in the production of better products for all end users [2]. Designs catering for older and younger individuals, will also satisfy commercial demand. According to the Office for National Statistics [3] the number of people in the United Kingdom aged over 60 has recently overtaken the number of those under 18 years of age, thus a product design that caters inclusively for all potential user age groups, will appeal to an ever-increasing commercial market.

\section{EXPERIMENTAL BACKGROUND}

Psychological research considers that the interactions individuals have with their environment are effectively learning processes, where the current situation is compared with that stored in memory (prior experience). If the situation is familiar this will ease the selection of an appropriate response (be it interactional or situational) and if novel, the situation may be analysed to discover any familiar aspects that may facilitate the selection of an appropriate response or increased understanding [4].

Other elements influence the effectiveness of interaction in conjunction with prior experience including Age and Generational Effects. Generational differences with regard to products and technology have been reported in literature and these differences are proposed to be symptomatic of exposure to technology at a particular stage in life [5, 6, and 7]. This may explain the difficulty older generations experience learning and interacting with various modern products and designs, which are often, compounded by the natural effects of ageing upon members the older population.

\section{COGNITIVE PROCESSING, MENTAL MODELS AND INTERACTION DESIGN}

During interactions individuals operate at differing levels of consciousness, and this can differ according to the familiarity of the situation. Rasmussen [8] proposed a model based on the assumption that individuals operated at a level appropriate to the familiarity of the situation, and an individual's previous experience of it, or of something similar to that situational experience. Wickens et al. [9] expanded this model to incorporate the types of processing that may occur - Skill, Rule and Knowledge based 
processing (Figure 1).

\begin{tabular}{|c|c|c|c|}
\hline \multirow[t]{2}{*}{$\begin{array}{l}\text { Familiarity / } \\
\text { Experience }\end{array}$} & & \multirow{3}{*}{$\begin{array}{l}\text { Rules - } \\
\text { If then assoc's } \\
\text { between } \\
\text { environmental } \\
\text { cues and actions } \\
\text { in memory }\end{array}$} & $\begin{array}{l}\text { Skill - } \\
\text { Minimal } \\
\text { cognitive } \\
\text { requirements due } \\
\text { to experience }\end{array}$ \\
\hline & & & \\
\hline \multirow[t]{2}{*}{$\begin{array}{c}\text { No } \\
\text { Familiarity / } \\
\text { Experience }\end{array}$} & $\begin{array}{l}\text { Knowledge - } \\
\text { Novel sibuations: } \\
\text { no stored rules, } \\
\text { cues, actions }\end{array}$ & & \\
\hline & $\begin{array}{l}\text { Conscious } \\
\text { Processing }\end{array}$ & & $\begin{array}{l}\text { Unconscious } \\
\text { Processing }\end{array}$ \\
\hline
\end{tabular}

Figure 1: Wickens' (1998) expanded version of Rasmussen's Skill, Rule and Knowledge-Based processing model

There are numerous theories proposed to explain the form and way in which information is perceived and encoded, and the extent to which such processing is a conscious activity. The connectionist approach considers that knowledge gained through experience is encoded and stored in memory in elements or nodes that form neural networks. When information in the environment is perceived nodes of the network associated with previous experience of this, or potentially similar perceptions, are activated and this activation spreads to other nodes. According to Johnson

[10] individuals experiences of the world consist of sensory- motor, emotional and social elements. Called image schemata, they represent internalised structures of human experience that guide and facilitate subsequentunderstanding. These schemata are considered not to be fixed, but flexible abstract patterns that are modified through further interaction with the world, in accordance with Norman's [11] views on mental models. Norman proposes that models are created by the accurate perception of a devices function and likely behaviour through its design. Thus, design may have a significant effect upon individuals' ability to perceive likely action or function, and thereby inhibit effective learning and interaction.

\section{RATIONALE FOR NOVEL PRODUCT INTERACTION}

The research aim was to determine what occurs during interaction with novel products about which users may possess limited prior experience or mental models - how learning occurs and how conceptualisations are developed through exposure. Do individuals identify familiar interface elements and combine them with experiential learning through
Skill, Rule and Knowledge- based reasoning, and are these internalised concepts facilitatory toward subsequent product interaction performance? Thus, a new-to-market, everyday high street product was used to minimise the extent of participants previous exposure. This provided the opportunity to investigate how users' mental models developed over time, exposure and experience. Other areas of interest to this research included the effects of natural atrophy and ageing, generational effects, and the effects of prior experience upon interaction (Figure 2).

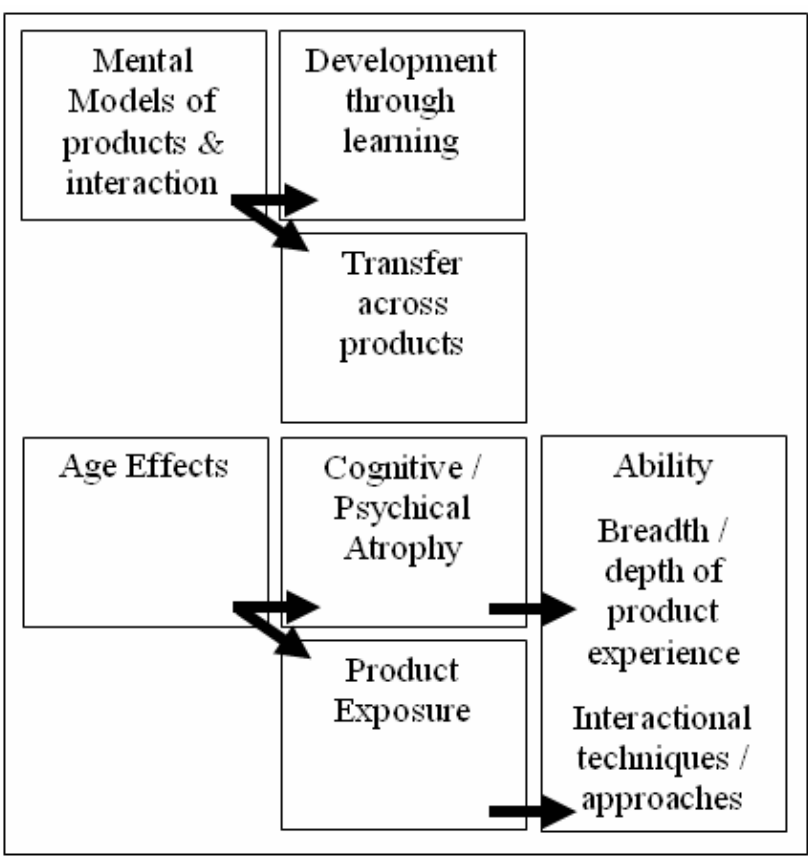

Figure 2: The scope of research

Presenting participants with the novel product and initially asking for their understanding of it allowed the identification of preconceptions held or initially developed. Having completed a number of tasks with the product whilst providing concurrent protocol, the initial questioning was duplicated at the midway stage and after further product exposure this was repeated during a post-interaction discussion. Overall this provided an opportunity to gain clarity on individuals developing understanding and internalised conceptualisations, and to ascertain if and how conceptualisations were modified through product exposure and interaction over time.

\section{SUMMARY OF PREVIOUS RESEARCH FINDINGS}

Thomas and van-Leeuwen [12], amongst others, concluded that similarity of prior experience to novel/ new scenarios is a determinant of performance and that there is an age-related decline in individual capability $[13,14]$. Further evidence was proposed for the generational effect - older users appearing 
reluctant or less able to complete tasks with modern technology products. These findings were supported by observations that older individuals exhibited a reluctance to invest in learning, and experienced difficulty modifying their mental models and approaches to problem-solving tasks. Furthermore, it has been noted that individuals' prior interactional experience is affected by the perception of product aesthetics and interactional complexity.

\section{RATIONALE FOR INCLUSIVE DESIGN}

Designing inclusively, with consideration of prior experience, can have a significant affect upon product acceptance, adoption, usability, perceived user-friendliness and commercial success [2]. Thus, the aim is not to create designs specifically for disenfranchised groups [15], but to focus on optimizing design for maximum accessibility in conjunction with minimizing the user effort required for interface or artifact interaction [16]. Thus, inclusive design has the commercial potential to increase long- term profits, enhance manufacturers' competitive edge, and can assist in the production of better products for all end users [2]. By studying how humans learn and interact with products and interfaces, it is the intention of this research to inform future design in terms of ease of use, ease of learning and understanding, and access to all [17].

\section{EXPERIMENTAL DESIGN \& METHOD}

\subsection{Sample}

A total of 30 individuals from a variety of backgrounds were recruited from outside academia to minimise any educational biases and maximise ecological validity. Participants were assigned to one of three groups according to age: $16-25$ (10 participants), 26-59 (10 participants) and 60-80 (10 participants). The total sample consisted of 18 males and 12 females.

\subsection{Tasks}

Participants were presented with the novel household product and requested to use it to complete 6 randomised tasks, the optimum interaction technique for which was determinable.

\subsection{Apparatus}

The apparatus consisted of the novel product a Plug-in Electricity Cost \& Usage Calculator (Figure 3 ) and an electrical device to be monitored - in this instance a desk lamp with a standard $60 \mathrm{w}$ bulb used for each trial.

A Sony Digital HandyCam was used to record participant verbalisations and interactional behaviour. Cantabeclipse TM software ensured all participants were sufficiently hand-eye coordinated and provided the opportunity to detect other agerelated differences. Technological Familiarity Questionnaires [7] and Standardised Interview Material were developed to ensure consistency between trials.

Participants underwent Cantabeclipse assessment, were initially presented with the product and then asked their understanding of it.

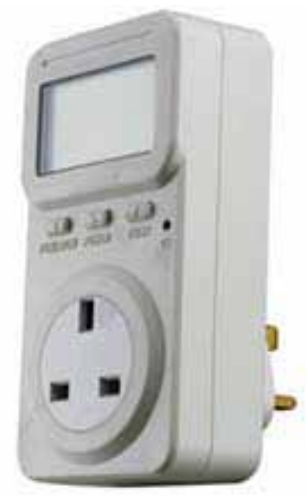

Figure 3: Plug-in Electricity Cost \& Usage Calculator

Having completed three tasks with the product, the discussion and questioning were repeated at the mid-way stage (Figure 4). After completing three final tasks, the questioning phase recommenced and was followed with the administration of a Technological Familiarity Questionnaire, based upon Blacklers [7] questionnaire. The questionnaire was used to verify participants' level of prior experience with various forms of technology products, and how frequently they interact with them.

Tasks 1-3 were randomly provided in the initial interaction phase, and 4-6 randomly provided in the subsequent interaction phase. Discussion in the post interaction phase centred upon participants' recognition of design metaphors present in the product, any perceived affordances within its design, any familiar features, and at what stage (if at all) the participants felt they understood the product and its interaction.

\begin{tabular}{cccc}
\hline $\begin{array}{c}\text { Stage 1 } \\
\text { Cantabeclipse } \\
\text { Asgessment }\end{array}$ & $\begin{array}{c}\text { Stage 2 } \\
\text { Initial } \\
\text { Exposare and } \\
\text { Product } \\
\text { Understanding }\end{array}$ & $\begin{array}{c}\text { Stage 3 } \\
\text { Completion of } \\
\text { Initial 3 Tasls }\end{array}$ & $\begin{array}{c}\text { Stage 4 } \\
\text { Subsequent } \\
\text { Product } \\
\text { Understanding }\end{array}$ \\
\hline $\begin{array}{c}\text { Stage 5 } \\
\text { Completion of } \\
\text { Final 3 Tasks }\end{array}$ & $\begin{array}{c}\text { Stage 6 } \\
\text { End Product } \\
\text { Understanding }\end{array}$ & $\begin{array}{c}\text { Stage 7 } \\
\text { Seni- } \\
\text { Stnctured } \\
\text { Interview }\end{array}$ & $\begin{array}{c}\text { Stage 8 } \\
\text { TFQ }\end{array}$ \\
& & Administration
\end{tabular}

Figure 4: Experimental procedure

The Cantabeclipse Cognitive Assessment Tool [8] offers neuropsychological assessment of capability, enabling rapid and accurate assessment regarding 
a wide range of central nervous system disorders. Although this was outside the main research area, it provided a useful screening mechanism that falls neatly within the scope of Inclusive Design research, as it possesses an integrated normative database to which subsequent participant performance can be compared. Two Cantabeclipse programs were chosen. The Motor Screening (MOT) test is intended to relax participants and introduce them to the bespoke interface (a touchscreen entry computer system) whilst screening for vision, movement and comprehension ability. The Spatial Span (SSP) test is a computerised version of the Corsi Block Task -a visuospatial analogue of the verbal-memory span task [9] and can be used to assess shortterm memory and neuropsychological impairment. Again, it is possible to subsequently compare scores according to age group membership. These facets are of significant peripheral interest to this study as memory in particular, is a key component in cognitive processing and the development of knowledge through learning.

\subsection{Experimental Design}

The experiment was 2-factor mixed design comparing people's performance and mental model development according to observation of their interaction with a novel device and their expressed knowledge of technology. The factors were:-

1. Between Subjects Factor:Age Group Membership (16-25, 26-59, 60-80) with 10 participants in each group

2. Within Subjects Factor was the completion of tasks (1-6)

The different aspects of performance and mental model development being measured as dependent variables were:

Task completion times, Number of Button Presses, Error Rate, Mean Time per Button Press, and Task Familiarity Questionnaire Score.

Participant verbalisations, interactional behaviour and semi- structured interview question answers were used to cross- reference both understanding and mental model development.

\subsection{Task Design}

The six tasks were presented as follows:

1. Find the lowest wattage reading for the device attached to the product.

2. Find the current reading for the device attached to the product.

3. Set Unit Cost Price to $99.50 £ / k W h$.
4. Find the frequency reading for the device attached to the product.

5. Find out how much the device attached to this product has consumed.

6 . Find the highest wattage reading for the device attached to the product.

The potential for order effects to influence performance was reduced by randomising the order in which tasks were provided. As Task 5 could only be achieved after completion of Task 3 , Task 3 was during the first half of the experimental procedure and Task 5 during the second half. The tasks remained numbered for identification purposes.

\subsection{Questionnaire Design}

Blackler's [7] Technological Familiarity Questionnaire was used, posing two questions about 15 contemporary technology products such as Mobile Phones and Ipods, and provided the following answer options:

When using the products, how many features of the product are you familiar with and do you use?

\begin{tabular}{|c|c|c|c|c|c|}
\hline 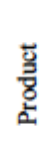 & 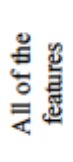 & 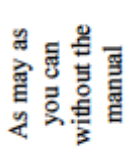 & 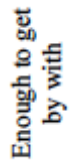 & 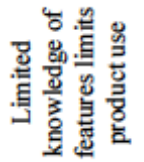 & 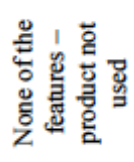 \\
\hline
\end{tabular}

How often do you use the following products?

\begin{tabular}{|c|c|c|c|c|c|c|c|}
\hline $\begin{array}{l}\overline{\mathrm{g}} \\
\text { के } \\
\text { 2. }\end{array}$ & $\begin{array}{l}\text { 胥 } \\
\text { 정 } \\
\text { 总 }\end{array}$ & 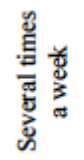 & 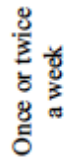 & 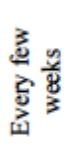 & $\begin{array}{l}\text { 总号 } \\
\text { 总高 } \\
\text { 总 }\end{array}$ & 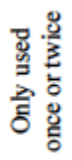 & $\begin{array}{l}\text { בे } \\
\text { ż }\end{array}$ \\
\hline
\end{tabular}

Responses were then rated according to Blackler's [7] protocol which then provided individual Question TFQ Scores and an overall combined TFQ score.

\subsection{Data Analysis and recording of elicited concurrent protocol}

Participants' interactional behaviour was recorded in close detail, allowing post-experimental analysis. Task completion times, total number of button presses, and button press error rates (the number of button presses made above the minimum required) were measured by reviewing the video-footage. The recording of the session allowed the notation of participants' verbal responses during interaction and particularly the pre, mid and postexperimental discussions. Verbalisations were noted in full and then analysed to extract information according to common themes, such as the quantification of the product concept - its purpose and operation as considered by participants initially, at the mid-way stage, and at the end.

Other points of interest were which product interface features, if any, were deemed familiar to participants, and from which products these features originated. 
Thus we attempted to determine any transference of knowledge from one product or interface to another. It was possible to formulate a list of frequently referenced products that either shared or possessed similar design features to also consider if feature familiarity facilitated learning, akin to the findings of Langdon et al [5].

\section{RESULTS}

\subsection{Interaction Data}

All interaction data were log transformed to stabilise the variance for the purposes of statistical analysis [20] and the data were analysed using a 2-way mixed design analysis of variance.

Task completion times. The older generation took longer to complete tasks than both younger age groups, with the 16-25 age group completing tasks in the quickest overall times (Graph 1).

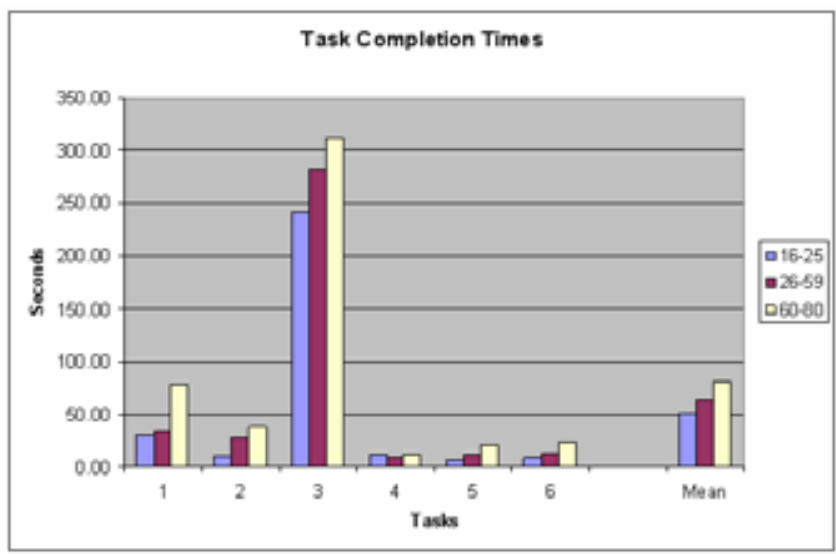

Graph 1: Task completion times

Within subject effect of task completion time: $F(5$, $135)=69.87, p<.01$ Between subjects factor of age group: $F(2,27)=7.153, p<.01$

Number of Button Presses. Graph 2 indicates that the older generation made a greater number of button presses toward task completion and the 1625 age group made the fewest.

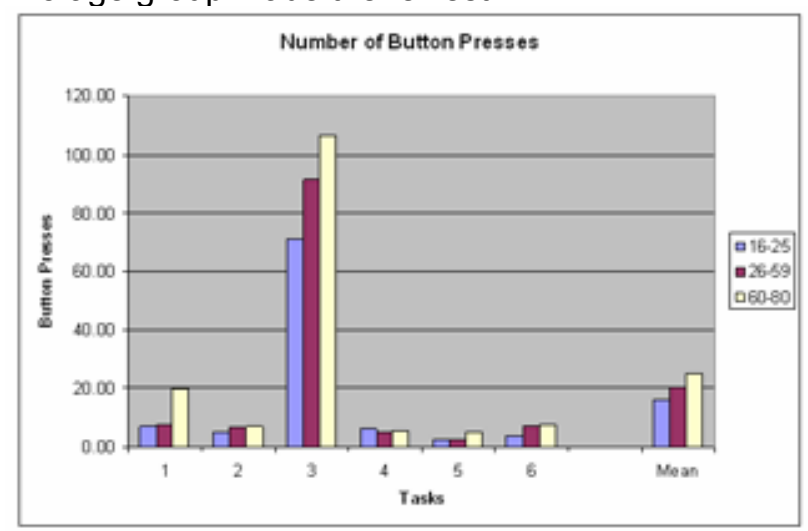

Graph 2: Button Press Data
Within subject effect of number of button presses: $F$ $(5,135)=75.409, p<.01$ Between subjects factor of age group: $F(2,27)=3.417, p<.05$

Error Rates. Error Rate data indicated that the older generation possessed a greater rate of error during product interaction, followed by the $26-59$ and 16 26 age groups respectively making fewer errors and exhibiting greater accuracy in their approaches (Graph 3).

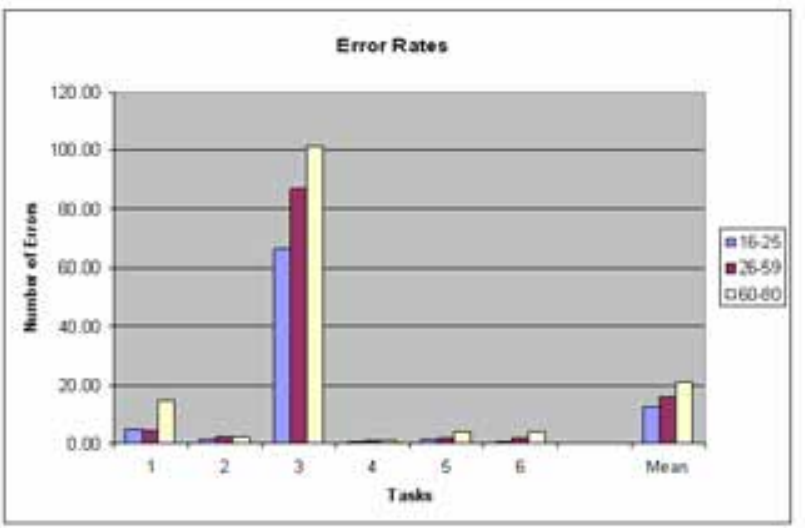

Graph 3: Error Rates

Within subject effect of error rate: $F(5,135)=87.833$, $p<.01$ Between subjects factor of age group: $F(2$, 27) $=3.44, p<.05$

Technological Familiarity Scores. With regard to Technological Familiarity, the 26-59 age group were most familiar with contemporary forms of technology; closely followed by the $16-25$ age group, with the older generation exhibiting the lowest TFQ scores (Graph 4).

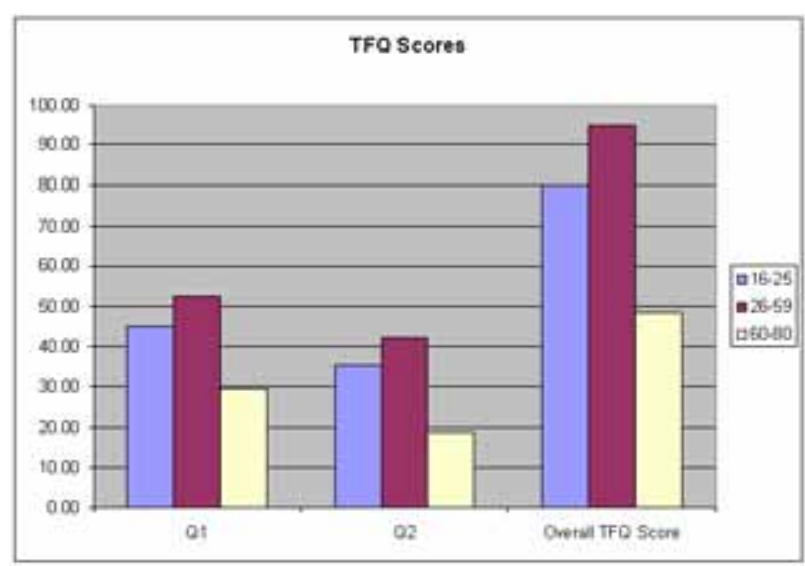

Graph 4: TFQ Score Comparison

Within subject effect of TFQ Score: $F(2,54)=$ 480.302, $p<.01$ Between subjects factor of Age Group: $F(2,27)=13.794, p<0.1$ 
Cantabeclipse SSP Results. The SSP Test designed to assess working memory capacity showed a similar trend per age group (Graph 5), the 26-59 age group remembering 7 items, the $16-25$ group 6.5 and the $60-80$ age group, 5 .

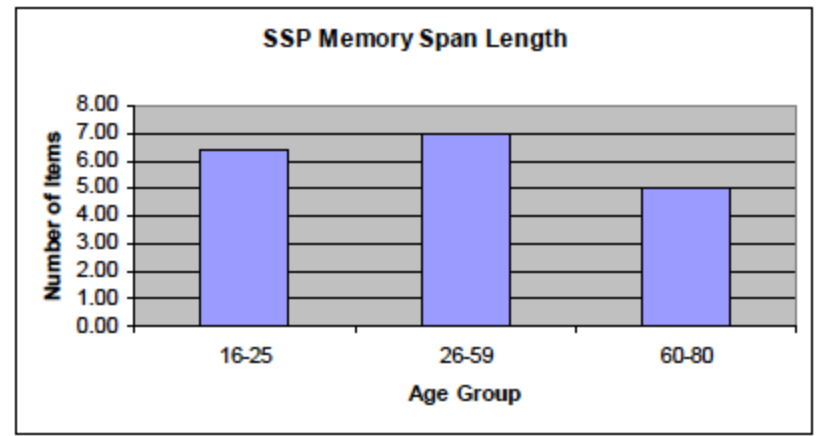

Graph 5: SSP Results

The results also suggested that no memory impairment was present in the older generation specifically, or in other age groups, due to natural atrophy or cognitive impairment. Indeed, the initial test (MOT) screening for vision, hearing, movement and comprehension impairment, highlighted no significant neuropsychological issues.

\subsection{Semi-structured Interview and Verbal Protocol Summary}

All participants initially recognised the product was electrical in nature, and the majority surmised that it was used as a measuring tool. Participants referenced plug-in devices in the initial phase of questioning and, if anything, it was evident that the 26-59 age group provided the most accurate and elaborate descriptions at this stage. By the midway stage participants had confirmed their original ideas and nearly all confirmed that it was designed to measure the flow of electricity, and that it could be set to indicate how much usage cost. The 6080 age group were the vaguest, having not (unlike other age groups) solidified their understanding of the product or its interaction at this stage. All age groups voiced disquiet at the complexity of setting the electrical cost function (Task 3 ). In the latter stage, the older generation provided the vaguest descriptions of the device, the 16-25 and 26-59 age groups providing more concrete, thorough, and accurate descriptions of the purpose, function and interaction of the product. The scrolling menu feature was learned and understood rapidly by all age groups, and was cited as being a design feature with which many were familiar. The most frequently cited product resemblance was to Digital Watches and Alarm Clocks, both featuring multi-button press requirements, scrolling menus and up and down adjustment controls.

\subsection{Overall Results Summary}

The younger generations made fewer button presses, are more accurate and their completion times are lower:

- Completion times: younger age groups perform quicker than older age groups

- Number of Button Presses: highest for older participants

- Error Rates lowest for younger age groups

- Technological Familiarity Scores highest for younger age groups

Overall, results as predicted may potentially be related to younger age groups possession of greater (prior) knowledge of current technology, and more accurate initial and subsequently rapid mental model development and enhancement.

\section{DISCUSSION}

The performance data indicates that with regard to task completion times, the younger generations exhibited faster responses and overall task completion times than the older generation (Graph 1). Indeed, the older generation took considerably longer to complete tasks in comparison with the other age groups. Although it may be that the older generation took more time to consider each move for a variety of reasons, it would appear they made more attempts and consequently made a greater proportion of erroneous attempts: this is supported by both the mean number of button press results (Graph 2) and the rate of error data (Graph 3). Observed in conjunction with each groups TFQ Scores, it is evident that the 26-59 age group possess the greatest awareness and level of interaction with contemporary technology. It appears a greater level of familiarity may correspond to a significant increase in overall task performance.

Conceptual development: In relation to interaction and learning, there is evidence of participants understanding a connection between both the effects of their interaction with the novel product and effects within their environment in accordance with Clark [16]. All participants were aware that the lamp influenced the energy monitor, or vice-versa. As all participants performed fewer button presses in the latter stages of the test than at the beginning, and made fewer references to not understanding how to proceed, it could be concluded that learning of interactional behaviour had occurred. In the latter 
stages the method of achieving task completion by repeatedly pressing the Function button was learnt in all conditions. This could be described as being performed automatically, verging on the Skilled or Rule Based levels of processing according to Wickens et al. [11] expanded model of Skill, Rule and Knowledge-based processing.

It appeared that the conceptual development of understanding or 'mental model' of the product occurred over the time-frame of exposure (Figure 5).

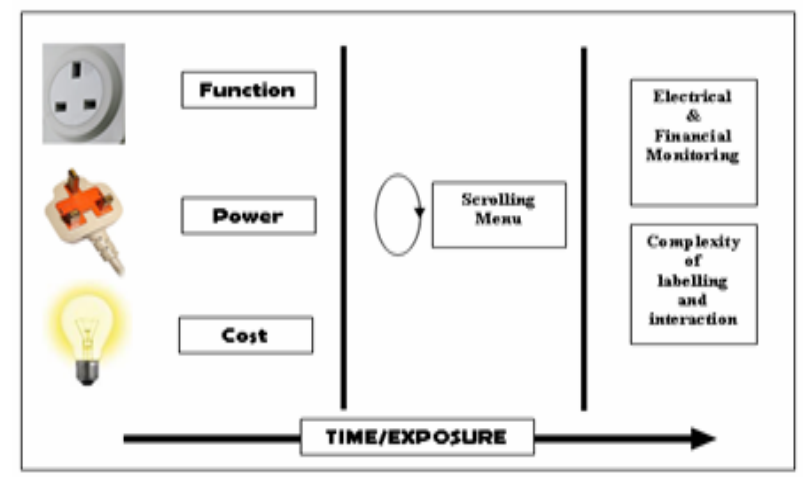

Figure 5: Conceptual evolution

Participants' rudimentary conception of the product and how it might function evolved over time, including initial recognition of the plug and socket configuration, and the artifacts relevance to electricity. Understanding the scrolling menu provided by repeated presses of the Function button appeared to have developed by the mid-way stage with overall understanding latterly complete. This is in accordance with Norman's [13] view that models are developed through experience, and are based on the perception of function and behaviour through design.

Inclusive design considerations.

With regard to the products design itself, there was an expectation of users that with so few buttons, the interaction of the device must be specific and, as it appeared to offer considerable functionality, complex. The utilisation of up and down arrows was recognised, almost universally, although accessing their function was not deemed intuitive. Generationally, a number of observations were made:

1. The display digits were considered large and assistive toward older individuals' perception. However, the units of measurement were deemed too small to ease recognition. Therefore, although the measurement was perceived, it was often indeterminable.

2. Colour could improve the products intuitive interaction, having the up and down arrows and square icon differentiated from the devices background would also assist their observation and the labels above buttons would be better discriminated had they been coloured.

3. Screen illumination was insufficient: in low lighting conditions, the display itself appeared difficult to read and this factor has high probability in the home with plug sockets at floor level.

4. An older participant explained that with increasing arthritis, they rarely felt the end of their fingertips, and thus, successfully manipulating the device was made increasingly awkward, given the button size.

5. Button design also limited the size, ease of recognition, and ease of finger-tip touch recognition of the icons embossed upon the buttons.

The interactional and observational evidence presented suggests that simple alterations to the physical design would enhance individuals' ability to learn and use this product. The specific results for Task 3 - setting the unit cost - also suggest an interactional design rethink may benefit all users in learning how to interact with this product, as difficulty in interaction was particularly manifest in this task regardless of age (Figure 6).

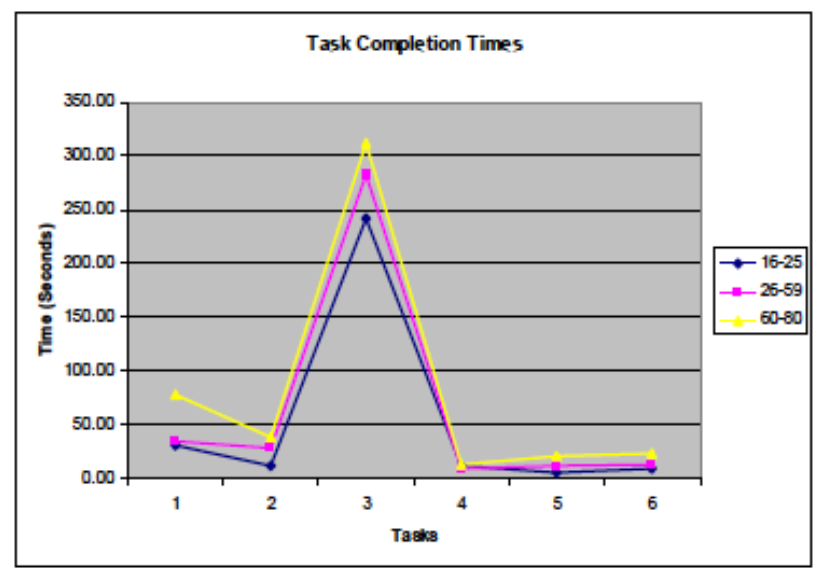

Figure 6: Comparison of Task Completion Times

Simplification of this procedure and maintaining similar levels of complexity to the interaction of the other available functions would reduce the level and extent of learning required when initially exposed to the product. The setting of the cost could have been improved by simply providing more effective visual, auditory or tactile feedback. It was however, clear that participants recognised consistent elements of the overall product concept, comprising of individual components, and outward aesthetic elements (Figure 7). It is therefore proposed that successful interaction occurs via the application of Skill, Rule and Knowledge-based reasoning [11], in conjunction with findings that remain inline with Norman's [13] views that internalisations are created and developed by the accurate perception of a devices function and likely behaviour through its design. 


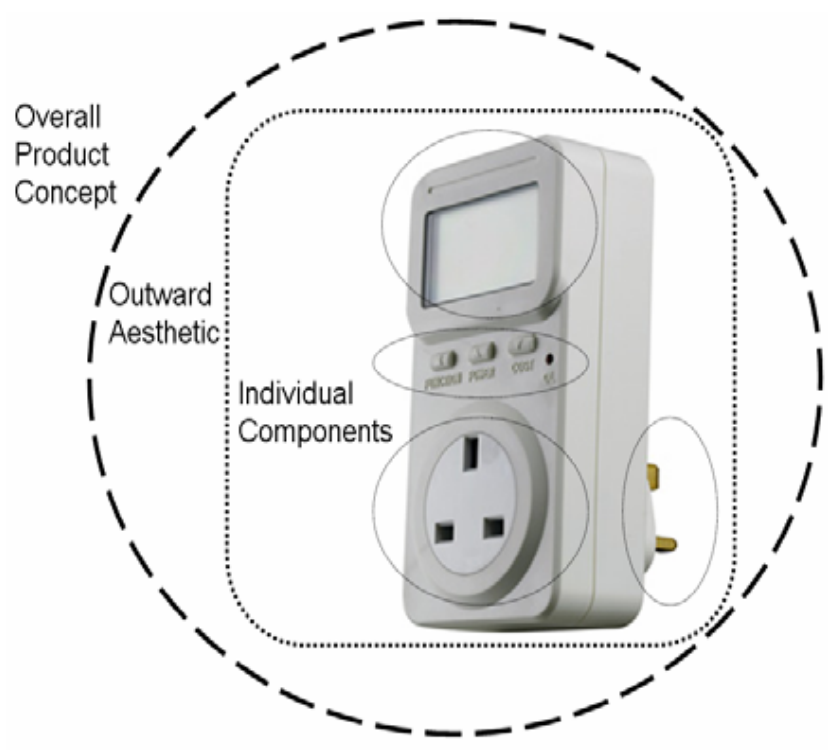

Figure 7: Overall product concept and constituent parts

\section{CONCLUSION}

Younger Generations completion times are lower and they exhibit a generally more accurate interaction approach. Older Generations appeared to adopt more rigid thinking and possess greater fear of error, inaccuracy and damaging the device and this may have contributed to reduced development of accurate product understanding.

"I would have thought you should only have
to press any of them once (the buttons)
not multiple times. You're afraid and think
pressing the buttons quickly will break it."

Participant (60-80 Age Group)

The trend is observed that with increasing age technological familiarity decreases. This may be due to an ageing effect - a reduced ability to recall useful/ relevant pieces of problem solving information, or due to a generational effect - the device may be more readily accepted and accessible to younger generations. The older generations lack of familiarity with contemporary and recent may provide further support for another generational effect - that as we age our inclination to 'keep up to date' with the latest developments may wean. Indeed, this was supported by further interview material:

\footnotetext{
"Young people would know about multi-button pressing and holding buttons, and have the patience to try different combinations, until they get the response they want. I just don't have the patience. I would try what I know, and if it didn't do what I wanted it to, l'd just go mad and give up with it." Participant (60-80 Age Group)
}

The dangers of design economy.

There are clearly financial and manufacturing reasons for producing products in the way this new to market energy monitor appears on the shelves. Ensuring a minimum number of components do a multitude of tasks may be cheaper initially. However, this economy may compromise usability as users struggle to engage with such products, promoting the sluggish adoption of technology by all users not only the older generation. Thus, in this instance, the economy of design does not translate well into simplicity, or user friendliness, of design. In fact, it appears to have produced the very opposite effect.

\section{FUTURE WORK}

The application of knowledge learned regarding prior experience and interaction will be transferred to other household products, devices and scenarios and attempt to predict user behaviour with other artefacts. Investigation into mental model development with a view to understand further how learning occurs and can be facilitated will also be continued. Ultimately an attempt will be made to verify ease of learning according to product features, and enhance understanding of Rasmussen's and Wicken's SRK model within learning and product interaction $[10,11]$. The overall intention is to propose a theory on how product interaction knowledge is acquired and how it is linked to performance and, through so doing, submit a design methodology that will maximise product adoption and ease of use to a larger proportion of the population and reduce the extent of age-related exclusion that currently occurs.

\section{ACKNOWLEDGMENTS}

The EPSRC is justly acknowledged in funding this research project, and our appreciation is also shown toward the Cambridge Network, CamCreative and the Cambridge Older Persons Enterprise (COPE) for their kind assistance.

\section{REFERENCES}

1. British Standards Institution: Design management systems. Managing inclusive design. BS 70006:2005. London: BSI (2005)

2. Dong, H., Bobjer, O., McBride, P. \& Clarkson, J.: Inclusive product design: Industrial case studies from the UK and Sweden. In P. Bust (Ed.), Contemporary Ergonomics (pp, 338-342). Taylor and Francis: Great Britain (2006)

3. Office for National Statistics. Last accessed March 2010, available from: http://news.bbc.co.uk/1/hi/ uk/7575869.stm

4. Edge, D., Blackwell, A., Dubuc, L.: The physical 
world as an abstract interface. In P. Bust (Ed.), Contemporary Ergonomics (pp. 224-228). Taylor and Francis: Great Britain (2006)

5. Langdon, P., Lewis, T., Clarkson, J.: The effects of prior experience on the use of consumer products. In: Universal Access in the Information Society, pp. 179-191. Springer, Heidelberg (2007)

6. Docampo-Rama, M.: Technology generations handling complex user interfaces. PhD Thesis. TU: Eindhoven (2001)

7. Blackler, A.: Intuitive interaction with complex artefacts. PhD Thesis. Queensland University of Technology: Australia. (2006)

8. Cantabeclipse (Cambridge Cognition). Last Accessed May 2010, available from: http://www. camcog.com/science/

9. Milner, B.: Interhemisphere difference in the localization of physiological processes in man. British Medical Bulletin, 27, 272-277. (1971)

10. Rasmussen, J.: Deciding and doing: Decision making in natural contexts. Norwood, NJ.: Ablex. (1993)

11. Wickens, C., Gordon, S., Liu, Y.: An introduction to human factors engineering. New York: AddisonWesley Educational Publishers Inc. (1998)

12. Johnson, M.: The body in the mind. The bodily basis of meaning, imagination, and reason. Chicago: The University of Chicago Press (1987)

13. Norman, D.: The design of everyday things. New York: Currency Doubleday. (1988)

14. Thomas, B., van-Leeuwen, M.: The user interface design of the fizz and spark GSM telephones. London: Taylor \& Francis (1999)

15. Wilkinson, C., Langdon, P., Clarkson J.: Investigating prior experience and product learning through novel interface interaction. Springer (2009) 16. Clark, A.: Being there. Putting brain, body, and world together again. Cambridge, MA.: MIT Press (1997)

17. Haigh, R.: The ageing process: a challenge for design, Applied Ergonomics, 24 (1), 9-14 (1993)

18. Deisinger, I., Breining, R., Robler, A., Holfe, I., Ruckert, D.: Immersive ergonomics analyses of console elements in a tractor cabin. In proceedings Fourth Immersive Projection Technologies Workshop, lowa: Ames, June 19-20, (2000)

19. Inclusive Design Group. Last accessed March 2010, available from: www-edc.eng.cam.ac.uk/ research/inclusivedesign/

20. Ericsson, K. (1974). Problem Solving behaviour with the 8Puzzle 1: Time to Solution. Report No. 431. Department of Psychology, University of Stockholm. 